\title{
About the Sign of Oriented Fredholm Operators between Banach Spaces
}

\author{
P. Benevieri, M. Furi, M. P. Pera and M. Spadini
}

\begin{abstract}
We give conditions for an oriented family of bounded Fredholm operators of index zero between Banach spaces to have a sign jump. In particular, we discuss criteria for detecting the sign jump in some special situations. For instance, when a sort of Crandall-Rabinowitz condition for bifurcation is assumed or in the case of a family of Leray-Schauder type. Finally, some examples of ordinary differential operators are presented to illustrate the meaning of the abstract results.
\end{abstract}

Keywords: Linear Fredholm operators, orientation, sign jump, Banach spaces

AMS subject classification: Primary 47A53, secondary 47E05, 34B05

\section{Introduction}

In [1] the first two authors introduced a fairly simple notion of orientation for linear Fredholm operators of index zero between real vector spaces. Any such operator, singular or non-singular, admits exactly two orientations, each of them making, by definition, the operator oriented. If an operator is invertible, one of the two possible orientations is more relevant than the other, and for this reason it is called natural (see Definition 3.4 below). Thus it makes sense to assign to any oriented isomorphism a sign: 1 if the orientation is natural and -1 in the opposite case. For a non-invertible Fredholm operator of index zero no one of the two orientations is more relevant than the other.

In the finite-dimensional context, the orientation of a linear operator turns out to be equivalent to the choice of a pair of orientations, one of the source space and one of the target space, up to an inversion of both of them. In this particular case the sign of an isomorphism agrees with the usual well-known

All authors: Dip. di Matem. Appl. 'G. Sansone', Via S. Marta 3, I-50139 Florence, Italy; benevieri@dma.unifi.it, furi@dma.unifi.it, pera@dma.unifi.it, spadini@dma.inifi.it

ISSN 0232-2064 / \$2.50 C Heldermann Verlag Berlin 
notion: 1 or -1 depending on whether the operator preserves or inverts the orientations of the spaces.

A crucial fact is that in the framework of Banach spaces the orientation has a sort of stability - in the sense that an orientation of an operator $L$ induces, in a very natural way, an orientation to any operator which is sufficiently close to $L$. Using this fact, the notion of orientation was extended (in $[1,2]$ ) to the nonlinear case; namely, to the case of a $C^{1}$ Fredholm map of index zero between real Banach spaces (and Banach manifolds). Such an extension coincides (in the $C^{1}$ case) with the notion given by Dold in [5: Exercise 6, p. 271] for maps between finite-dimensional manifolds and, in the most important cases, with the notion due to Fitzpatrick, Pejsachowicz and Rabier in [9] for maps between Banach manifolds. The definition in [9], however, does not agree completely in the finite-dimensional case with that in [5]. For example, a constant map, whose domain is a non-orientable manifold, is non-orientable according to Dold and orientable according to Fitzpatrick, Pejsachowicz and Rabier (but, peculiarly, with only one orientation).

In [1], by means of the concept of orientation, a degree theory for Fredholm maps between Banach manifolds was introduced. This degree agrees, in the most important cases, with that developed in [6 - 9]. The difference between the two theories, however, is mainly in the construction method and, in our opinion, in the simplicity of the definition given in [1] which easily leads to a homotopy invariance property in the $C^{1}$ case.

Let $f: M \rightarrow N$ be an oriented Fredholm map of index zero between two real Banach manifolds and let $y \in N$ be such that $f^{-1}(y)$ is compact. In this case the triple $(f, M, y)$ is said to be admissible for the degree (see [1]). Assume first that $(f, M, y)$ is generic; that is, $y$ is a regular value for $f$. According to [1], the degree in this case is, as in the finite-dimensional context, an algebraic count of the number of solutions of the equation $f(x)=y$. More precisely,

$$
\operatorname{deg}(f, M, y)=\sum_{x \in f^{-1}(y)} \operatorname{sign}\left(f^{\prime}(x)\right)
$$

where $f^{\prime}(x): T_{x} M \rightarrow T_{y} N$ is the Fréchet derivative of $f$ at $x$, and $\operatorname{sign}\left(f^{\prime}(x)\right)$ denotes the sign of the oriented isomorphism $f^{\prime}(x)$. In the general case the degree is defined by considering the restriction of $f$ to a convenient neighborhood $U$ of $f^{-1}(y)$ and putting

$$
\operatorname{deg}(f, M, y)=\operatorname{deg}(f, U, z)
$$

where $z$ is a regular value for $f$ which is sufficiently close to $y$.

To compute the degree of an admissible triple, the usual technique is the following: first, if necessary, deform $(f, M, y)$, via a suitable homotopy, to 
a simpler admissible triple $(g, M, z)$, where $z$ is a regular value for $g$; then compute

$$
\sum_{x \in g^{-1}(z)} \operatorname{sign}\left(g^{\prime}(x)\right) .
$$

It is therefore of some interest to develop methods for evaluating the sign of an oriented isomorphism. That is, methods to decide whether or not the orientation of a given oriented isomorphism coincides with the natural one. This is, in fact, our purpose.

As we shall see, a practical way of orienting a Fredholm operator of index zero $L$ is, when possible, to transport the orientation of a selected, naturally oriented isomorphism $S$ along the line segment joining $S$ and $L$. In this case, if $L$ is invertible, one gets $\operatorname{sign}(L)=(-1)^{n}$, where $n$ is the number of sign jumps along the segment. This formula, as we shall prove, is in the spirit of LeraySchauder theory, where the index of an invertible linear compact perturbation of the identity $L=I-K$ is $(-1)^{n}$, where $n$ is the number of characteristic values of $K$ in $(0,1)$ with odd algebraic multiplicity. Having this in mind, we shall give some results for detecting the sign jumps of a one-parameter family $\left\{L_{\lambda}\right\}$ of Fredholm linear operators of index zero.

Another motivation for giving conditions ensuring the existence of a sign jump of a family $\left\{L_{\lambda}\right\}$ as above is related to bifurcation theory. In fact, let $E$ and $F$ be real Banach spaces and let $f: \mathbb{R} \times E \rightarrow F$ be continuous and continuously differentiable with respect to the second variable. Assume that the partial derivative $\partial_{2} f(\lambda, x)$ is Fredholm of index zero for any $(\lambda, x)$. As proven in $[3,13]$, if $f(\lambda, 0)=0$ for all $\lambda \in \mathbb{R}$ and the family $L_{\lambda}=\partial_{2} f(\lambda, 0)$ has a sign jump at some $\lambda_{0}$, then $\lambda_{0}$ is a bifurcation point for the equation $f(\lambda, x)=0$. In addition, if $f$ is proper on any bounded closed subset of $\mathbb{R} \times E$, a sign jump at $\lambda_{0}$ implies the existence of a connected set of nontrivial solution pairs $(\lambda, x)$, i.e. with $x \neq 0$, whose closure contains $\left(\lambda_{0}, 0\right)$ and is either unbounded or meets the $\lambda$-axis outside $\left(\lambda_{0}, 0\right)$.

\section{Preliminaries}

Given two vector spaces $E$ and $F$, we shall denote by $\mathrm{L}(E, F)$, or simply by $\mathrm{L}(E)$ when $F=E$, the vector space of linear operators from $E$ into $F$. By $\mathrm{F}(E, F)$, or $\mathrm{F}(E)$ when $F=E$, we will mean the subspace of $\mathrm{L}(E, F)$ of the operators with finite-dimensional image. The image of an element $L \in \mathrm{L}(E, F)$ will be denoted by $\operatorname{Im} L$, its kernel by $\operatorname{Ker} L$, and its cokernel (i.e. the quotient $F / \operatorname{Im} L$ ) by coKer $L$. Other useful notations are $\operatorname{Iso}(E, F)$ for the set of isomorphisms from $E$ onto $F$, and $\mathrm{GL}(E)$ for the set of the automorphisms of $E$ (i.e. $\mathrm{GL}(E)=\operatorname{Iso}(E, E)$ ). 
Let us recall some useful properties of Fredholm operators between vector spaces.

A linear operator $L: E \rightarrow F$ is (algebraic) Fredholm if Ker $L$ and coKer $L$ are finite-dimensional. In this case, its index is the integer

$$
\begin{aligned}
\operatorname{ind}(L) & =\operatorname{dim}(\operatorname{Ker} L)-\operatorname{codim}(\operatorname{Im} L) \\
& =\operatorname{dim}(\operatorname{Ker} L)-\operatorname{dim}(\operatorname{coKer} L)
\end{aligned}
$$

The set of Fredholm operators of index $n$ between $E$ and $F$ will be denoted by $\Phi_{n}(E, F)$.

Observe that, given a vector space $E$ and a finite-codimensional subspace $E_{1}$ of $E$, the inclusion $S: E_{1} \rightarrow E$ is Fredholm with $\operatorname{ind}(S)=-\operatorname{codim}\left(E_{1}\right)$, and any projection $P: E \rightarrow E_{1}$ (onto $E_{1}$ ) is Fredholm with $\operatorname{ind}(S)=$ $\operatorname{codim}\left(E_{1}\right)$.

Remark 2.1. If $E$ and $F$ are finite-dimensional, then any linear operator from $E$ into $F$ is Fredholm of index $\operatorname{dim}(E)-\operatorname{dim}(F)$. This is a consequence of the well-known formula

$$
\operatorname{dim}(E)=\operatorname{dim}(\operatorname{Ker} L)+\operatorname{dim}(\operatorname{Im} L)
$$

The following useful result is a direct consequence of the definition of Fredholm operator. Recall first that, given a linear operator $L: E \rightarrow F$, a subspace $F_{1}$ of $F$ is said to be transverse to $L$ if $\operatorname{Im} L+F_{1}=F$.

Lemma 2.2. Let $L: E \rightarrow F$ be a Fredholm operator and let $F_{1}$ be a subspace of $F$ which is transverse to $L$. Then the restriction

$$
L_{1}: L^{-1}\left(F_{1}\right) \rightarrow F_{1}
$$

of $L$ is Fredholm with $\operatorname{ind}\left(L_{1}\right)=\operatorname{ind}(L)$.

The following lemma is useful for computing the index of a Fredholm operator written in block-matrix form. For its proof we refer to [10: Part 1, Chapter 3, Section 1].

Lemma 2.3. Assume $E=E_{1} \oplus E_{2}$ and $F=F_{1} \oplus F_{2}$, and write $a$ Fredholm operator $L: E \rightarrow F$ in the block-matrix form $L=\left(\begin{array}{ll}A & B \\ C & D\end{array}\right)$. If $A: E_{1} \rightarrow F_{1}$ is invertible, then $\operatorname{ind}(L)=\operatorname{ind}\left(D-C A^{-1} B\right)$.

The following result is the algebraic analogy of a well-known one in the context of Banach spaces (see statement (ii) in Theorem 2.6 below). The simple proof is given for the sake of completeness. 
Theorem 2.4. Let $L: E \rightarrow F$ be Fredholm, and take $K \in \mathrm{F}(E, F)$. Then the operator $L+K$ is Fredholm and $\operatorname{ind}(L+K)=\operatorname{ind}(L)$.

Proof. Let $F_{1}$ be a finite-dimensional subspace of $F$ containing $\operatorname{Im} K$ and transverse to $L$. Put $E_{1}=L^{-1}\left(F_{1}\right)$. Clearly, $E_{1}$ is finite-dimensional. In fact, because of Lemma 2.2 and Remark 2.1, one has $\operatorname{dim}\left(E_{1}\right)-\operatorname{dim}\left(F_{1}\right)=\operatorname{ind}(L)$. Let $E_{0}$ be such that $E_{0} \oplus E_{1}=E$, and define $F_{0}=L\left(E_{0}\right)$. It is easy to check, using the transversality of $F_{1}$, that $F=F_{0} \oplus F_{1}$. Writing $L$ and $K$ in block-matrix form, we get

$$
L=\left(\begin{array}{cc}
L_{00} & 0 \\
0 & L_{11}
\end{array}\right) \quad \text { and } \quad L+K=\left(\begin{array}{cc}
L_{00} & 0 \\
K_{10} & L_{11}+K_{11}
\end{array}\right) .
$$

Thus $L+K$ is Fredholm, $L_{00}: E_{0} \rightarrow F_{0}$ being an isomorphism. Lemma 2.3 implies the following two equalities:

$$
\operatorname{ind}(L)=\operatorname{ind}\left(L_{11}\right) \quad \text { and } \operatorname{ind}(L+K)=\operatorname{ind}\left(L_{11}+K_{11}\right) .
$$

The assertion now follows from Remark 2.1

Theorem 2.5. Let $L_{1}: E_{1} \rightarrow E_{2}$ and $L_{2}: E_{2} \rightarrow E_{3}$ be Fredholm operators. Then $L_{2} L_{1}$ is Fredholm and $\operatorname{ind}\left(L_{2} L_{1}\right)=\operatorname{ind}\left(L_{1}\right)+\operatorname{ind}\left(L_{2}\right)$.

Proof. See, e.g., the proof of Theorem 13.1 in [14: Chapter IV] which is still valid in the purely algebraic context

We pass now to recall some useful properties of Fredholm operators in the framework of Banach spaces. In this category, whose objects are Banach spaces, the morphisms are bounded linear operators. Therefore, from now on, all the linear operators between Banach spaces will be assumed continuous. In this particular context, the spaces $\mathrm{L}(E, F)$ and $\mathrm{F}(E, F)$, and the sets Iso $(E, F), \mathrm{GL}(E)$ and $\Phi_{n}(E, F)$ will be considered made up of bounded operators only. Any direct sum in a Banach space is assumed to be topological (besides being algebraic); that is, the summands are closed subspaces of the containing space.

Fredholm operators between Banach spaces enjoy many properties and are studied in detail by several authors (see, e.g., [14]). We state two important properties that are meaningful in this context.

Theorem 2.6. Let $E$ and $F$ be Banach spaces. The following properties hold:

(i) The set $\Phi_{n}(E, F)$ is open in the Banach space $\mathrm{L}(E, F)$.

(ii) If $K \in \mathrm{L}(E, F)$ is compact and $L \in \Phi_{n}(E, F)$, then $L+K \in \Phi_{n}(E, F)$.

We conclude this section by recalling that if $L: E \rightarrow F$ is a Fredholm operator between Banach spaces, then its image is necessarily a closed subspace of $F$. Moreover, the set of surjective operators between $E$ and $F$ (not necessarily Fredholm) is open in $\mathrm{L}(E, F)$. 


\section{Oriented Fredholm operators and oriented maps}

In this section we first summarize (with some minor changes) the notion of orientation for a Fredholm operator of index zero between real vector spaces introduced in [1]. After that we show how such a notion becomes stable in the context of Banach spaces. Then we give a brief review of the concept of orientability for a continuous family of (bounded) Fredholm operators of index zero between Banach spaces. We refer to [1 - 3] for more details.

The concept of orientation for a Fredholm operator of index zero is purely algebraic. The basic tool to define such a notion is a simple extension of the determinant to the infinite-dimensional context. Let us recall first this extension.

Let $T: E \rightarrow E$ be an endomorphism of a (not necessarily real) vector space and denote by $I$ the identity operator on $E$. Assume that the image of the operator $K=I-T$ is contained in a finite-dimensional subspace $E_{1}$ of $E$. Thus $T$ maps $E_{1}$ into itself and, consequently, the determinant of its restriction $T_{1}: E_{1} \rightarrow E_{1}$ is well defined. As shown in [1], this determinant does not depend on the space $E_{1}$ containing $\operatorname{Im} K$. Therefore, it makes sense to call determinant of $T$ this common value, and to denote it by $\operatorname{det}(T)$. From Theorem 2.4 it follows that $T$ is Fredholm of index zero. Moreover, one can easily check that, as in the case when $E$ is finite-dimensional, $T$ is invertible if and only if $\operatorname{det}(T) \neq 0$.

From now on, given a vector space $E$, the affine subspace of $\mathrm{L}(E)$ of the operators that are admissible for the determinant will be denoted by $\Psi(E)$. Namely,

$$
\Psi(E)=\{T \in \mathrm{L}(E): I-T \in \mathrm{F}(E)\} .
$$

The proof of the following useful result is a routine task.

Proposition 3.1. The determinant has the following fundamental properties:

(1) If $T \in \Psi(E)$ and $E_{1}$ is any subspace of $E$ containing $\operatorname{Im}(I-T)$, then the restriction $T_{1}$ of $T$ to $E_{1}$ belongs to $\Psi\left(E_{1}\right)$ and $\operatorname{det}(T)=\operatorname{det}\left(T_{1}\right)$.

(2) If $T_{1}, T_{2} \in \Psi(E)$, then $T_{2} T_{1} \in \Psi(E)$ and $\operatorname{det}\left(T_{2} T_{1}\right)=\operatorname{det}\left(T_{2}\right) \operatorname{det}\left(T_{1}\right)$.

(3) If $S: F \rightarrow E$ is an isomorphism and $T \in \Psi(E)$, then $S^{-1} T S \in \Psi(F)$ and $\operatorname{det}\left(S^{-1} T S\right)=\operatorname{det}(T)$.

(4) If $T_{1} \in \Psi\left(E_{1}\right)$ and $T_{2} \in \Psi\left(E_{2}\right)$, then $T_{1} \times T_{2} \in \Psi\left(E_{1} \times E_{2}\right)$ and $\operatorname{det}\left(T_{1} \times T_{2}\right)=\operatorname{det}\left(T_{1}\right) \operatorname{det}\left(T_{2}\right)$.

In many cases, a practical method for computing the determinant of an operator $T \in \Psi(E)$ is given by the following consequence of Proposition 3.1. 
Corollary 3.2. Let $T \in \mathrm{L}(E)$ and let $E=E_{0} \oplus E_{1}$. Assume that, with this decomposition of $E$, the matrix representation of $T$ is of the type

$$
\left(\begin{array}{ll}
I_{0} & U \\
V & S
\end{array}\right)
$$

where $I_{0}$ is the identity operator on $E_{0}$. If $\operatorname{dim}\left(E_{1}\right)<+\infty$ (or, more generally, if $S \in \Psi\left(E_{1}\right)$ and the operators $U$ and $V$ have finite-dimensional image), then $T \in \Psi(E)$ and

$$
\operatorname{det}(T)=\operatorname{det}(S-V U) \text {. }
$$

Proof. Recall that, given the decomposition $E=E_{0} \oplus E_{1}$, the entries of the matrix representation of an operator $R \in \mathrm{L}(E)$ are defined as $P_{j} R S_{i} \quad(i, j=0,1)$ where $S_{0}: E_{0} \rightarrow E$ and $S_{1}: E_{1} \rightarrow E$ denote the inclusions, and $P_{0}: E \rightarrow E_{0}$ and $P_{1}: E \rightarrow E_{1}$ are the projections associated with the decomposition. Taking into account this, it is easy to show that $T \in \Psi(E)$. Hence $\operatorname{det}(T)$ makes sense.

Observe first that equality (3.1) is true in the particular case of a lower triangular matrix; that is, when $U$ is the trivial operator. In this case, in fact, $I-T$ maps $E$ into $E_{1}$, therefore $\operatorname{det}(T)=\operatorname{det}(S)$, since $S$ is the restriction of $T$ to $E_{1}$ (use property (1) of Proposition 3.1 if $\operatorname{dim}\left(E_{1}\right)=+\infty$ ).

The assertion is also true for the upper triangular matrix $R=\left(\begin{array}{cc}I_{0} & -U \\ 0 & I_{1}\end{array}\right)$ where $I_{1}$ denotes the identity on $E_{1}$. To show this, it is enough to consider the restriction of $R$ to any finite-dimensional space containing $E_{1}+\operatorname{Im} U$.

To prove the assertion in the general case, consider the composite operator $T R$. Given $x_{0} \in E_{0}$ and $x_{1} \in E_{1}$, with a standard computation one gets

$$
T R\left(x_{0}+x_{1}\right)=x_{0}+\left(V x_{0}+S x_{1}-V U x_{1}\right) .
$$

Therefore $T R$ can be represented by the triangular matrix $\left(\begin{array}{cc}I_{0} & 0 \\ V & S-V U\end{array}\right)$. Thus, $\operatorname{det}(T R)=\operatorname{det}(S-V U)$. On the other hand, property (2) of Proposition 3.1 implies $\operatorname{det}(T R)=\operatorname{det}(T) \operatorname{det}(R)$ and, consequently, $\operatorname{det}(T)=\operatorname{det}(S-V U)$, as claimed

Let now $E$ and $F$ be two real vector spaces and let $L \in \Phi_{0}(E, F)$. A linear operator $A: E \rightarrow F$ is a corrector of $L$ if its image is finite-dimensional and $L+A$ is an isomorphism. Denote by $\mathcal{C}(L)$ the set of correctors of $L$. It is easy to see that $C(L)$ is non-empty. Indeed, any linear operator $A \in \mathrm{L}(E, F)$ such that $\operatorname{Ker} A \oplus \operatorname{Ker} L=E$ and $\operatorname{Im} A \oplus \operatorname{Im} L=F$ is a corrector of $L$. In particular, if $L$ is an isomorphism, the trivial operator belongs to $\mathcal{C}(L)$.

We define an equivalence relation on $\mathcal{C}(L)$ as follows. Let $A, B \in \mathcal{C}(L)$ and consider the endomorphism of $E$

$$
T=(L+B)^{-1}(L+A)=I-(L+B)^{-1}(B-A) .
$$


Clearly, $T$ is an invertible finite-dimensional perturbation of the identity. Thus, its determinant is defined and non-zero. We say that $A$ is equivalent to $B$ or, more precisely, $A$ is $L$-equivalent to $B$, if

$$
\operatorname{det}\left((L+B)^{-1}(L+A)\right)>0 .
$$

In [1] it is shown that this is an equivalence relation on $\mathcal{C}(L)$ with two equivalence classes. A simpler proof of this could be carried out as a straightforward consequence of Proposition 3.1.

Definition 3.3. Let $L$ be a Fredholm operator of index zero between real vector spaces. An orientation of $L$ is one of the two equivalence classes of $\mathcal{C}(L)$. The operator $L$ is oriented when one of its orientations is selected.

Formally, an oriented operator is a pair $L=\left(L^{\prime}, \omega\right)$, where $L^{\prime} \in \Phi_{0}(E, F)$ and $\omega$ is one of the two equivalence classes of $\mathcal{C}\left(L^{\prime}\right)$. However, for the sake of simplicity, unless required for the understanding, we shall not use different symbols to discern between an oriented operator $L$ and its underlying nonoriented part $L^{\prime}$.

As in [2], the set of all oriented operators from $E$ into $F$ will be denoted by $\widehat{\Phi}_{0}(E, F)$, or simply by $\widehat{\Phi}_{0}(E)$ when $F=E$. Observe that there is a natural projection

$$
p: \widehat{\Phi}_{0}(E, F) \rightarrow \Phi_{0}(E, F)
$$

defined by forgetting the orientation.

Given $L \in \widehat{\Phi}_{0}(E, F)$, its orientation is denoted by $\mathcal{C}_{+}(L)$ and called class of positive correctors of $L$. The other equivalence class is the opposite orientation of $L$ and denoted by $\mathcal{C}_{-}(L)$. The elements of $\mathcal{C}_{-}(L)$ are the negative correctors of $L$.

When an operator $L$ is invertible, a distinguished orientation emerges.

Definition 3.4 (Natural orientation). An oriented isomorphism $L: E \rightarrow$ $F$ has the natural orientation if the trivial operator is a positive corrector of $L$.

Let $L \in \Phi_{0}(E, F)$. Given an isomorphism $S: G \rightarrow E$, from property (3) of Proposition 3.1 one can immediately deduce that two correctors $A$ and $B$ of $L$ are $L$-equivalent if and only if $A S$ and $B S$ are $L S$-equivalent. An analogous assertion holds for a composition of the type $S L$, with $S \in \operatorname{Iso}(F, G)$. This justifies the following

Definition 3.5. Let $L_{1} \in \widehat{\Phi}_{0}\left(E_{1}, E_{2}\right)$ and $L_{2} \in \widehat{\Phi}_{0}\left(E_{2}, E_{3}\right)$ be two oriented operators, and denote by $L_{1}^{\prime}$ and $L_{2}^{\prime}$ the corresponding non-oriented operators. The oriented composition $L_{2} L_{1}$ is the composition $L_{2}^{\prime} L_{1}^{\prime}$ with 
the orientation obtained by choosing as a positive corrector any operator $K \in \mathrm{F}\left(E_{1}, E_{3}\right)$ of the type

$$
K=\left(L_{2}^{\prime}+A_{2}\right)\left(L_{1}^{\prime}+A_{1}\right)-L_{2}^{\prime} L_{1}^{\prime}
$$

with $A_{1} \in \mathcal{C}_{+}\left(L_{1}\right)$ and $A_{2} \in \mathcal{C}_{+}\left(L_{2}\right)$.

Notice that the oriented composition is associative. Thus, it makes sense to consider the oriented composition of three, or even more, oriented operators. Unless otherwise specified, from now on, the composition of oriented operators will be regarded as an oriented composition.

The orientation of an operator $L \in \Phi_{0}(E, F)$ can be regarded as the orientation of the restriction of $L$ to any suitable pair of subspaces of $E$ and $F$. Precisely, let $F_{1}$ be a subspace of $F$ that is transverse to $L$ and let $E_{1}=$ $L^{-1}\left(F_{1}\right)$. Consider any direct complement $E_{0}$ to $E_{1}$ in $E$ and split $E$ and $F$ as $E=E_{0} \oplus E_{1}$ and $F=L\left(E_{0}\right) \oplus F_{1}$. With this decomposition, $L$ can be represented by a matrix $\left(\begin{array}{cc}L_{0} & 0 \\ 0 & L_{1}\end{array}\right)$. Clearly, $L_{0}$ is an isomorphism and $L_{1} \in$ $\Phi_{0}\left(E_{1}, F_{1}\right)$. Thus, any linear operator $A: E \rightarrow F$ of the type

$$
\left(\begin{array}{ll}
0 & 0 \\
0 & A_{1}
\end{array}\right)
$$

is a corrector of $L$ if and only if $A_{1}$ is a corrector of $L_{1}$. From Proposition 3.1 one can easily deduce that two correctors of $L_{1}$ are $L_{1}$-equivalent if and only if the corresponding correctors of $L$ (according to (3.2)) are $L$-equivalent. This shows that the following definition is well posed.

Definition 3.6. Given $L \in \Phi_{0}(E, F)$, let $F_{1}$ be a subspace of $F$ which is transverse to $L$, and denote by $L_{1}$ the restriction of $L$ to $L^{-1}\left(F_{1}\right)$ as domain and to $F_{1}$ as codomain. Two orientations, of $L$ and $L_{1}$ respectively, are said to be correlated (or one induced by the other) if there exists a projection $P$ of $E$ onto $L^{-1}\left(F_{1}\right)$ and a positive corrector $A_{1}$ of $L_{1}$ such that the operator $A=S A_{1} P$ is a positive corrector of $L$, where $S: F_{1} \rightarrow F$ is the inclusion. If $L$ and $L_{1}$ are oriented with correlated orientations, $L_{1}$ is called the oriented restriction of $L$ and $L$ the oriented extension of $L_{1}$.

We define now the sign of an oriented operator.

Definition 3.7. Given $L \in \widehat{\Phi}_{0}(E, F)$, its sign is the integer

$$
\operatorname{sign}(L)= \begin{cases}+1 & \text { if } L \text { is invertible and naturally oriented } \\ -1 & \text { if } L \text { is invertible and not naturally oriented } \\ 0 & \text { if } L \text { is not invertible. }\end{cases}
$$

Remark 3.8. Let $L \in \Phi_{0}(E, F)$ and let $A$ be a corrector of $L$. We have

$$
(L+A)^{-1} L=I-(L+A)^{-1} A .
$$


Thus $(L+A)^{-1} L$ is a finite-dimensional perturbation of the identity and, consequently, its determinant is well defined. One can easily check that if $L$ is oriented and $A$ is a positive corrector of $L$, then

$$
\operatorname{sign}(L)=\operatorname{sign}\left(\operatorname{det}\left((L+A)^{-1} L\right)\right) .
$$

From the notion of correlated orientations (Definition 3.6) one can easily deduce the following property of the sign.

Proposition 3.9 (Reduction Property). Let $L \in \Phi_{0}(E, F)$ and let $F_{1}$ be a subspace of $F$ that is transverse to $L$. Denote by $L_{1}$ the restriction of $L$ to the spaces $L^{-1}\left(F_{1}\right)$ and $F_{1}$. If $L$ and $L_{1}$ are oriented with correlated orientations, then

$$
\operatorname{sign}(L)=\operatorname{sign}\left(L_{1}\right) .
$$

Another useful property of the sign is expounded by the following proposition, whose proof is immediate.

Proposition 3.10 (Invariance Property). Let $L \in \widehat{\Phi}_{0}(E, F)$, and let $S: G \rightarrow E$ and $T: F \rightarrow H$ be two naturally oriented isomorphisms. Then

$$
\operatorname{sign}(T L S)=\operatorname{sign}(L) .
$$

From now on, unless otherwise specified, all the vector spaces considered are real Banach spaces, and all the linear operators between them are assumed to be bounded. Therefore, given any (bounded) $L \in \Phi_{0}(E, F)$, by a corrector of $L$ we shall actually mean a bounded corrector. By abuse of notation, the set of correctors of $L$ will be still denoted by $\mathcal{C}(L)$, even if it is a subset of the set considered in the purely algebraic case. As a consequence of the HahnBanach Theorem, the set $\mathcal{C}(L)$ is non-empty also in this enriched context, and an orientation of $L$ can be regarded as an equivalence class of bounded correctors of $L$. As in the algebraic case, the set of oriented operators from a Banach space $E$ into a Banach space $F$ will be denoted by $\widehat{\Phi}_{0}(E, F)$, even if, we recall, all the operators are assumed to be bounded.

The salient fact that distinguishes the orientation in the context of Banach spaces from the orientation in the algebraic case is, loosely speaking, the influence exerted by an oriented operator over its neighbors. More precisely, due to the fact that $\operatorname{Iso}(E, F)$ is open in $\mathrm{L}(E, F)$, any corrector of a given $L \in \Phi_{0}(E, F)$ remains a corrector for all the operators in a neighborhood of $L$. This means that an oriented operator induces, by a sort of stability, an orientation on any sufficiently close operator. Thus, it is evident how to endow $\widehat{\Phi}_{0}(E, F)$ with a topology which makes the natural projection

$$
p: \widehat{\Phi}_{0}(E, F) \rightarrow \Phi_{0}(E, F)
$$

a two-fold covering space (see [2] for a formal definition). 
In the context of Banach spaces one may ask whether or not the determinant function det $: \Psi(E) \rightarrow \mathbb{R}$ is continuous. The following example provides a negative answer.

Example 3.11. For any $n \in \mathbb{N}$, let $T_{n}: \ell^{2} \rightarrow \ell^{2}$ be the operator obtained by multiplying the first $n$ coordinates of any $x \in \ell^{2}$ by $1+\frac{1}{n}$ (and leaving unchanged all the others). Clearly, $T_{n} \in \Psi\left(\ell^{2}\right)$. Moreover,

$$
\operatorname{det}\left(T_{n}\right)=\left(1+\frac{1}{n}\right)^{n}
$$

since $1+\frac{1}{n}$ is the only eigenvalue different from 1 and its multiplicity is $n$. Thus $\operatorname{det}\left(T_{n}\right) \rightarrow e$. On the other hand, $T_{n} \rightarrow I$ and $\operatorname{det}(I)=1$.

Notice that, in the above example, the images of the operators $I-T_{n}$ are not equicontained in a finite-dimensional subspace of $\mathrm{L}\left(\ell^{2}\right)$. In fact, when this happens, the determinant is continuous. More precisely, as a straightforward consequence of the definition of determinant, one gets the following result.

Lemma 3.12. Let $E_{1}$ be a finite-dimensional subspace of a Banach space $E$. Then the restriction of the determinant function to the affine subspace

$$
\left\{T \in \mathrm{L}(E): \operatorname{Im}(I-T) \in E_{1}\right\}
$$

of $\Psi(E)$ is continuous.

The following useful result shows that, in spite of fact that the determinant function can be discontinuous, its sign, if non-zero, is stable.

Proposition 3.13. Let $E$ be a Banach space. Then the restriction of the function $T \mapsto \operatorname{sign}(\operatorname{det}(T))$ to the open subset $\mathrm{GL}(E) \cap \Psi(E)$ of $\Psi(E)$ is continuous.

Proof. Let $T_{0} \in \mathrm{GL}(E) \cap \Psi(E)$. Since $\mathrm{GL}(E)$ is open, there exists a convex neighborhood $U$ of $T_{0}$ in $\Psi(E)$ contained in $\operatorname{GL}(E)$. Thus $\operatorname{det}(T) \neq 0$ for all $T \in U$. Let us show that

$$
\operatorname{sign}(\operatorname{det}(T))=\operatorname{sign}\left(\operatorname{det}\left(T_{0}\right)\right)
$$

for all $T \in U$. Given any $T_{1} \in U$, let $E_{1}$ be a finite-dimensional subspace of $E$ containing both $\operatorname{Im}\left(I-T_{0}\right)$ and $\operatorname{Im}\left(I-T_{1}\right)$. Thus, for any $\lambda \in[0,1]$, the operator

$$
T_{\lambda}=(1-\lambda) T_{0}+\lambda T_{1}
$$

is such that $\operatorname{Im}\left(I-T_{\lambda}\right) \subseteq E_{1}$. On the basis of Lemma 3.12 this implies that the function $\lambda \mapsto \operatorname{det}\left(T_{\lambda}\right)$ is continuous. The result now follows since, for any $\lambda \in[0,1], T_{\lambda}$ belongs to $U$ and, consequently, $\operatorname{det}\left(T_{\lambda}\right) \neq 0$ 
We are ready to introduce the concept of orientation for a continuous family of Fredholm operators of index zero.

Definition 3.14. Let $\Lambda$ be a topological space and $h: \Lambda \rightarrow \Phi_{0}(E, F)$ be a continuous map. An orientation of $h$ is a continuous choice of an orientation $\omega(\lambda)$ of $h(\lambda)$ for each $\lambda \in \Lambda$, where "continuous" means that for any $\lambda \in \Lambda$ there exists $A_{\lambda} \in \omega(\lambda)$ such that $A_{\lambda} \in \omega(\mu)$ for all $\mu$ in a neighborhood of $\lambda$. The map $h$ is orientable when it admits an orientation and oriented when an orientation has been chosen. In particular, a subset $\mathcal{A}$ of $\Phi_{0}(E, F)$ is said to be orientable (or oriented) if so is the inclusion $i: \mathcal{A} \rightarrow \Phi_{0}(E, F)$.

Notice that if $\mathcal{A} \subseteq \Phi_{0}(E, F)$ is orientable, then so is any subset of $\mathcal{A}$ and, more generally, any continuous map $h: \Lambda \rightarrow \mathcal{A}$. In fact, an orientation of $\mathcal{A}$ induces an orientation on any map $h: \Lambda \rightarrow \mathcal{A}$.

An outstanding (and somehow surprising) result of Kuiper (see [11]) asserts that the subset $\mathrm{GL}\left(\ell^{2}\right)$ of $\Phi_{0}\left(\ell^{2}\right)$ is contractible. In particular, it is connected. As shown in [2], this implies that $\Phi_{0}\left(\ell^{2}\right)$ is not orientable.

Perhaps the simplest example of a non-constant orientable map $h: \Lambda \rightarrow$ $\Phi_{0}(E, F)$ is when any $h(\lambda)$ is invertible. To show this, endow $h$ with the natural orientation, namely the orientation given by choosing the trivial operator as a positive corrector of $h(\lambda)$ for any $\lambda \in \Lambda$. Observe that in this case one has $\operatorname{sign}(h(\lambda))=1$ for all $\lambda \in \Lambda$.

Clearly, any orientable map $h$ admits at least two orientations. In fact, if $h$ is oriented by $\omega$, reverting this orientation at any $\lambda \in \Lambda$, one gets what we call the opposite orientation $\omega_{-}$of $h$.

Remark 3.15. As a consequence of Proposition 3.13, one can easily deduce that if $A$ and $B$ are two equivalent correctors of a given $L_{0} \in \Phi_{0}(E, F)$, then they remain $L$-equivalent for any $L$ in a neighborhood of $L_{0}$. This implies that the notion of "continuous choice of an orientation" in Definition 3.14 is equivalent to the following:

- For any $\lambda \in \Lambda$ and any $A_{\lambda} \in \omega(\lambda)$, there exists a neighborhood $U$ of $\lambda$ such that $A_{\lambda} \in \omega(\mu)$ for all $\mu \in U$.

Remark 3.15 implies that the set in which two orientations of $h$ coincide is open in $\Lambda$, and for the same reason it is open the set in which two orientations of $h$ are opposite one to the other. Therefore, if $\Lambda$ is connected, the map $h$, if orientable, admits exactly two orientations. As a straightforward consequence of this argument (or, if one prefers, of Proposition 3.13) one obtains the following result, which is a sort of intermediate value theorem.

Proposition 3.16. Let $h: \Lambda \rightarrow \Phi_{0}(E, F)$ be an oriented map defined on a connected space $\Lambda$. Assume there are two points $\lambda_{1}, \lambda_{2} \in \Lambda$ such that

$$
\operatorname{sign}\left(h\left(\lambda_{1}\right)\right) \operatorname{sign}\left(h\left(\lambda_{2}\right)\right)<0 .
$$


Then there exists $\lambda_{0} \in \Lambda$ such that $\operatorname{sign}\left(h\left(\lambda_{0}\right)\right)=0$.

We observe that the notion of "continuous choice of an orientation" in Definition 3.14 becomes the usual concept of continuity if one regards the orientation of the map $h: \Lambda \rightarrow \Phi_{0}(E, F)$ as a lifting $\hat{h}: \Lambda \rightarrow \widehat{\Phi}_{0}(E, F)$ of $h$ in the covering space

$$
p: \widehat{\Phi}_{0}(E, F) \rightarrow \Phi_{0}(E, F) .
$$

The advantage of this point of view is that one could use known results on covering space theory to deduce important properties of orientable maps, such as the homotopy invariance of the orientability, the transport of the orientation by means of a homotopy, the orientability and non-orientability of some maps (see [2] for details).

In particular, from covering space theory one gets that if $\Lambda$ is simply connected and locally path connected, then any continuous map $h: \Lambda \rightarrow$ $\Phi_{0}(E, F)$ is orientable (see [2]). Hence, in this case, $h$ admits exactly two orientations, each of them is uniquely determined by the orientation of $h$ at any chosen $\lambda_{0} \in \Lambda$.

As a consequence of this, any convex subset of $\Phi_{0}(E, F)$ is orientable. More generally, given $S \in \Phi_{0}(E, F)$, let Star $S$ denote the union of all convex subsets of $\Phi_{0}(E, F)$ containing $S$. Clearly, an operator $L \in \mathrm{L}(E, F)$ belongs to Star $S$ if and only if the line segment joining $S$ and $L$ is contained in $\Phi_{0}(E, F)$. Consequently, since $\Phi_{0}(E, F)$ is open in $\mathrm{L}(E, F)$, so is $\operatorname{Star} S$. Thus, Star $S$ is simply connected (being star-shaped) and locally path connected (being open). In particular, it is orientable with just two orientations. Taking this into account, the following definition provides a practical way to assign an orientation to a large class of orientable subsets of $\Phi_{0}(E, F)$.

Definition 3.17. Given $S \in \operatorname{Iso}(E, F)$ and $L \in \mathrm{L}(E, F)$, we say that $L$ is oriented by $S$ (or $S$-oriented, for short) if the line segment $\overline{S L}$ joining $S$ and $L$ is contained in $\Phi_{0}(E, F)$ and $L$ is oriented according to the orientation of $\overline{S L}$ determined by the natural orientation of $S$. A subset $\mathcal{A}$ of $\operatorname{Star} S$ is $S$-oriented if so is any $L \in \mathcal{A}$. More generally, an orientation of a map $h: \Lambda \rightarrow \operatorname{Star} S$ is an $S$-orientation if any $h(\lambda)$ is $S$-oriented.

The following is a simple and important example of a set oriented by the identity.

Example 3.18 (Leray-Schauder case). Let $\Psi_{K}(E)$ denote the compact hull of the identity operator in a Banach space $E$; that is,

$$
\Psi_{K}(E)=\{L \in \mathrm{L}(E): I-L \in K(E)\}
$$

where $K(E)$ is the subspace of $\mathrm{L}(E)$ of the compact linear operators. Then $\Psi_{K}(E)$ is a convex subset of Star $I$ (actually, an affine subspace of $\mathrm{L}(E)$ ) and, 
therefore, can be (and will be, unless otherwise specified) oriented by the identity.

It is not difficult to check that, between the two orientations of $\mathrm{L}\left(\mathbb{R}^{n}\right)=$ $\operatorname{Star}(I)$, the $I$-orientation is the one satisfying the condition

$$
\operatorname{sign}(L)=\operatorname{sign}(\operatorname{det}(L)) \quad\left(L \in \mathrm{L}\left(\mathbb{R}^{n}\right)\right)
$$

Clearly, the opposite orientation is such that

$$
\operatorname{sign}(L)=-\operatorname{sign}(\operatorname{det}(L)) \quad\left(L \in \mathrm{L}\left(\mathbb{R}^{n}\right)\right) .
$$

More generally, as a consequence of Corollary 5.2 (see Remark 5.3) we will show that, given any $I$-oriented automorphism $L \in \Psi_{K}(E)$, its sign equals its Leray-Schauder degree.

\section{Finite dimensional reduction}

Let $J \subseteq \mathbb{R}$ be an open interval, and consider an oriented family $\left\{L_{\lambda}\right\}_{\lambda \in J}$ of Fredholm operators of index zero between two Banach spaces $E$ and $F$; that is, an oriented map from $J$ into $\Phi_{0}(E, F)$. Assume there exists $\lambda_{0} \in J$ such that

$$
L_{\lambda} \text { is an isomorphism for any } \lambda \in J \backslash\left\{\lambda_{0}\right\} \text {. }
$$

We are interested in sufficient (and necessary) conditions for $L_{\lambda}$ to have a sign jump at $\lambda=\lambda_{0}$.

From now on, without loss of generality, we shall assume $\lambda_{0}=0$ and $L_{0}$ singular, the last assumption being necessary for the existence of a sign jump (because of Proposition 3.16).

Let $F_{0}$ be a Banach space and let $P: F \rightarrow F_{0}$ and $Q: F \rightarrow \mathbb{R}^{n}$ be bounded linear operators with the following properties:

$P$ and $Q$ are surjective

Ker $Q \oplus \operatorname{Ker} P=F$

$P L_{0}$ is surjective.

We observe that such a pair of operators, $P$ and $Q$, always exists. For example, one could take as $P$ any projection of $F$ onto $F_{0}=\operatorname{Im} L_{0}$ (or onto a finitecodimensional subspace $F_{0}$ of $\left.\operatorname{Im} L_{0}\right)$ and as $Q$ the associated projection $I-P$ onto the space Ker $P$, which can be regarded as $\mathbb{R}^{n}$ for some $n \in \mathbb{N}$. 
Remark 4.1. Properties (4.1a) - (4.1b) above hold if and only if the operator $P \times Q: y \mapsto(P y, Q y)$ is an isomorphism between $F$ and $F_{0} \times \mathbb{R}^{n}$.

Remark 4.2. Property (4.1c) holds if and only if $P$ is surjective and $L_{0}$ is transverse to $\operatorname{Ker} P$.

Remark 4.3. Since $Q$ is surjective, $\operatorname{Ker} Q$ has codimension $n$. Hence, from (4.1b) it follows $\operatorname{dim} \operatorname{Ker} P=n$. Thus $P$, being surjective, is Fredholm of index $n$. Therefore, $P L_{\lambda}$ is Fredholm and

$$
\operatorname{ind}\left(P L_{\lambda}\right)=\operatorname{ind}(P)+\operatorname{ind}\left(L_{\lambda}\right)=n .
$$

Moreover, since $P L_{0}$ is surjective, so is $P L_{\lambda}$ for $\lambda$ small. Consequently, for these values of $\lambda$ one has $\operatorname{dim}\left(\operatorname{Ker} P L_{\lambda}\right)=n$.

Given $P$ and $Q$ as above, the space $F$ can be regarded as the direct sum of two fixed subspaces: $\operatorname{Ker} Q$ and $\operatorname{Ker} P$. The fact that $P L_{0}$ is surjective allows us to define a $\lambda$-dependent splitting of $E$ in two subspaces in such a way that $L_{\lambda}$ can be represented, up to the composition with isomorphisms, as a triangular $2 \times 2$ block-matrix.

Let $A_{\lambda}: \mathbb{R}^{n} \rightarrow E$, with $\lambda \in J$ sufficiently small, be a continuous family of isomorphisms between $\mathbb{R}^{n}$ and Ker $P L_{\lambda}$. (An explicit construction of such a family is given in Lemma 4.5 below.) Let $E_{0}$ be a direct complement to Ker $P L_{0}$ in $E$. Clearly, for small values of $\lambda$, the map

$$
B_{\lambda}: E_{0} \times \mathbb{R}^{n} \rightarrow E
$$

given by $\left(u_{0}, u_{1}\right) \mapsto u_{0}+A_{\lambda} u_{1}$ is an isomorphism and depends continuously on $\lambda$. Let

$$
C_{\lambda}: E_{0} \times \mathbb{R}^{n} \rightarrow F_{0} \times \mathbb{R}^{n}
$$

be the composition $(P \times Q) L_{\lambda} B_{\lambda}$, which can be regarded as a representation of $L_{\lambda}$, up to the isomorphisms $B_{\lambda}$ and $(P \times Q)$. Since $P L_{\lambda} A_{\lambda}=0$, writing $C_{\lambda}$ in block-matrix form, we get

$$
C_{\lambda}=\left(\begin{array}{cc}
\left.P L_{\lambda}\right|_{E_{0}} & 0 \\
\left.Q L_{\lambda}\right|_{E_{0}} & Q L_{\lambda} A_{\lambda}
\end{array}\right) .
$$

Remark 4.4. Since $L_{\lambda}$ is an isomorphism for small $\lambda \neq 0$, so is the endomorphism $Q L_{\lambda} A_{\lambda}$ of $\mathbb{R}^{n}$.

The following result, applied to $M_{\lambda}=P L_{\lambda}$, ensures the existence of the family $A_{\lambda}$ required in the above construction. 
Lemma 4.5. Let $M_{\lambda}: E \rightarrow F_{0} \quad(\lambda \in J)$ be a continuous family of linear operators between Banach spaces. Assume that $M_{0}$ is surjective with $n$-dimensional kernel $(n \in \mathbb{N})$. Then, for $\lambda$ small enough, there exists a continuous family of injective linear operators $A_{\lambda}: \mathbb{R}^{n} \rightarrow E$ such that any $A_{\lambda}$ maps $\mathbb{R}^{n}$ onto $\operatorname{Ker} M_{\lambda}$.

Proof. Let $A_{0}: \mathbb{R}^{n} \rightarrow E$ be an isomorphism between $\mathbb{R}^{n}$ and $\operatorname{Ker} M_{0}$, and let $E_{0}$ be a (closed) complement to $\operatorname{Ker} M_{0}$ in $E$. The operator from $E_{0} \times \mathbb{R}^{n}$ to $E$ defined by $\left(u_{0}, u_{1}\right) \mapsto u_{0}+A_{0} u_{1}$ is an isomorphism. Fix any $\lambda \in J$ and let $u \in \operatorname{Ker} M_{\lambda}$ be given. There exist (and are unique) $u_{0} \in E_{0}$ and $u_{1} \in \mathbb{R}^{n}$ such that $u=u_{0}+A_{0} u_{1}$. One has

$$
0=M_{\lambda} u=M_{\lambda} u_{0}+M_{\lambda} A_{0} u_{1} .
$$

Since $\left.M_{0}\right|_{E_{0}}$ is an isomorphism between $E_{0}$ and $F_{0}$, the same is true for $\left.M_{\lambda}\right|_{E_{0}}$ when $\lambda$ is small. Thus, by (4.2),

$$
\begin{aligned}
u_{0} & =-\left(\left.M_{\lambda}\right|_{E_{0}}\right)^{-1} M_{\lambda} A_{0} u_{1} \\
u & =A_{0} u_{1}-\left(\left.M_{\lambda}\right|_{E_{0}}\right)^{-1} M_{\lambda} A_{0} u_{1} .
\end{aligned}
$$

For small $\lambda$, define $A_{\lambda}: \mathbb{R}^{n} \rightarrow E$ by

$$
A_{\lambda} x=A_{0} x-\left(\left.M_{\lambda}\right|_{E_{0}}\right)^{-1} M_{\lambda} A_{0} x .
$$

Clearly, $A_{\lambda}$ depends continuously on $\lambda$, and the notation is consistent with the initial choice of $A_{0}$ since $M_{0} A_{0}=0$.

From (4.2) - (4.3) one gets $\operatorname{Ker} M_{\lambda} \subseteq \operatorname{Im} A_{\lambda}$. Clearly, $\operatorname{dim} \operatorname{Im} A_{\lambda} \leq n$. Since, for $\lambda$ small, $M_{\lambda}$ is onto and Fredholm of index $n$, one has $\operatorname{dim} \operatorname{Ker} M_{\lambda}=$ $n$. Thus $\operatorname{Ker} M_{\lambda}=\operatorname{Im} A_{\lambda}$, which implies the assertion

The following is the main result of this section.

Theorem 4.6. Let $\left\{L_{\lambda}\right\}_{\lambda \in J}, P: F \rightarrow F_{0}$ and $Q: F \rightarrow \mathbb{R}^{n}$ be as above. Let $A_{\lambda}: \mathbb{R}^{n} \rightarrow E$ be a continuous family of injective linear operators such that $\operatorname{Im} A_{\lambda}=\operatorname{Ker} P L_{\lambda}$. Then $L_{\lambda}$ has a sign jump at $\lambda=0$ if and only if the same is true for the function $\lambda \mapsto \operatorname{det}\left(Q L_{\lambda} A_{\lambda}\right)$.

Proof. We will deduce the assertion from the reduction and invariance properties of oriented operators (see Propositions 3.9 and 3.10).

As above, let $E_{0}$ be such that $E=E_{0} \oplus \operatorname{Ker} P L_{0}$ and define $B_{\lambda}: E_{0} \times$ $\mathbb{R}^{n} \rightarrow E$ by $B_{\lambda}\left(u_{0}, u_{1}\right)=u_{0}+A_{\lambda} u_{1}$. Endow the isomorphisms $B_{\lambda}$ and $P \times Q$ with the natural orientation. Recalling that $\left\{L_{\lambda}\right\}_{\lambda \in J}$ is an oriented family, consider the oriented composition

$$
C_{\lambda}=(P \times Q) L_{\lambda} B_{\lambda}: E_{0} \times \mathbb{R}^{n} \rightarrow F_{0} \times \mathbb{R}^{n} .
$$


By the Invariance Property of the sign (Proposition 3.10),

$$
\operatorname{sign}\left(C_{\lambda}\right)=\operatorname{sign}\left(L_{\lambda}\right) .
$$

Since $P L_{0}$ is surjective, so is $P L_{\lambda}$ for $\lambda$ small. Therefore, for these values of $\lambda, C_{\lambda}$ is transverse to the subspace $\{0\} \times \mathbb{R}^{n}$ of $F_{0} \times \mathbb{R}^{n}$.

Now, observe that $C_{\lambda}^{-1}\left(\{0\} \times \mathbb{R}^{n}\right)$ coincides, for any $\lambda$, with the subspace $\{0\} \times \mathbb{R}^{n}$ of $E_{0} \times \mathbb{R}^{n}$. Consequently, by the Reduction Property (Proposition 3.9), the sign of $C_{\lambda}$, for $\lambda$ small, coincides with the sign of the oriented restriction $\widehat{C}_{\lambda}$ of $C_{\lambda}$ to the subspace $\{0\} \times \mathbb{R}^{n}$ of $E_{0} \times \mathbb{R}^{n}$, as domain, and to the subspace $\{0\} \times \mathbb{R}^{n}$ of $F_{0} \times \mathbb{R}^{n}$, as codomain.

Clearly, the operator $\widehat{C}_{\lambda}$ can be canonically identified with the endomorphism $Q L_{\lambda} A_{\lambda}$ of $\mathbb{R}^{n}$, whose induced orientation, without loss of generality, can be assumed to be the $I$-orientation. Indeed, the existence of a sign jump does not depend on the chosen orientation of $\left\{L_{\lambda}\right\}_{\lambda \in J}$. Thus, with this assumption, one has

$$
\operatorname{sign}\left(C_{\lambda}\right)=\operatorname{sign}\left(Q L_{\lambda} A_{\lambda}\right)=\operatorname{sign}\left(\operatorname{det}\left(Q L_{\lambda} A_{\lambda}\right)\right),
$$

and the assertion follows from (4.4)

In some applications it may happen that the choice of one of the operators, $P$ or $Q$, is quite natural. It is useful to have criteria which allow, given $P$, to construct $Q$ so that properties (4.1) are satisfied, and vice versa.

Assume we are given $P: F \rightarrow F_{0}$ such that

(1) $P L_{0}$ is surjective

(2) Ker $P$ has finite dimension, say $n$.

In this case, we can define $Q$ by composing any projection onto $\operatorname{Ker} P$ with any isomorphism from Ker $P$ to $\mathbb{R}^{n}$.

Assume now we are given $Q: F \rightarrow \mathbb{R}^{n}$ such that:

(1) $Q$ is surjective.

(2) There exists a subspace $F_{1}$ of $F$ such that $\operatorname{Ker} Q \oplus F_{1}=F$ and $F_{1}$ is transverse to $\operatorname{Im} L_{0}$. (For example, this is true when $\operatorname{Ker} Q \subseteq \operatorname{Im} L_{0}$.)

In this case we can take $P$ as the projector onto $F_{0}=\operatorname{Ker} Q$ associated with the decomposition $\operatorname{Ker} Q \oplus F_{1}=F$.

We observe that, given an oriented family $\left\{L_{\lambda}\right\}_{\lambda \in J}$ as above, one can always split both the spaces $E$ and $F$ in two subspaces, say $E_{0}, E_{1} \subseteq E$ and $F_{0}, F_{1} \subseteq F$, in order to express $L_{\lambda}$ as $\left(\begin{array}{cc}T_{\lambda} & U_{\lambda} \\ V_{\lambda} & S_{\lambda}\end{array}\right)$ with $E_{1}$ and $F_{1}$ finitedimensional, and $T_{0}$ invertible. For example, take $E_{0}$ a (closed) complement to $\operatorname{Ker} L_{0}, E_{1}=\operatorname{Ker} L_{0}, F_{0}=\operatorname{Im} L_{0}$, and $F_{1}$ a complement to $F_{0}$. Since $E_{1}$ and $F_{1}$ must have the same dimension (say $n$ ), up to isomorphisms we may suppose $E_{1}=F_{1}=\mathbb{R}^{n}$. With this in mind, we state the following consequence of Theorem 4.6. 
Theorem 4.7. Let $L_{\lambda}: E \rightarrow F \quad(\lambda \in J)$ be as above, and assume $E=E_{0} \times \mathbb{R}^{n}$ and $F=F_{0} \times \mathbb{R}^{n}$, with $E_{0}$ and $F_{0}$ Banach spaces. Let $\left(\begin{array}{ll}T_{\lambda} & U_{\lambda} \\ V_{\lambda} & S_{\lambda}\end{array}\right)$ be the matrix representation of $L_{\lambda}$ according to the above decompositions of $E$ and $F$. If $T_{0}$ is invertible, then $L_{\lambda}$ has a sign jump at $\lambda=0$ if and only if the same happens to the real function $\lambda \mapsto \operatorname{det}\left(S_{\lambda}-V_{\lambda} T_{\lambda}^{-1} U_{\lambda}\right)$.

Proof. Let $P$ and $Q$ denote the two canonical projections of $F=F_{0} \times \mathbb{R}^{n}$ onto the factors $F_{0}$ and $\mathbb{R}^{n}$, respectively. Observe that

$$
\operatorname{Ker} P L_{\lambda}=\left\{\left(x_{0}, x_{1}\right) \in E_{0} \times \mathbb{R}^{n}: T_{\lambda} x_{0}+U_{\lambda} x_{1}=0\right\}
$$

coincides with the image of the injective operator $A_{\lambda}: \mathbb{R}^{n} \rightarrow E_{0} \times \mathbb{R}^{n}$ given by $A_{\lambda} x_{1}=\left(-T_{\lambda}^{-1} U_{\lambda} x_{1}, x_{1}\right)$, which is defined for $\lambda$ small because $T_{0}$ is invertible. Thus

$$
Q L_{\lambda} A_{\lambda}=S_{\lambda}-V_{\lambda} T_{\lambda}^{-1} U_{\lambda}
$$

and the assertion follows from Theorem 4.6

\section{Special cases}

In this section we discuss criteria for detecting a sign jump in particular situations. Although the cases considered look quite different, the construction presented in the previous section provides a general framework.

As before, $E$ and $F$ denote real Banach spaces, $J$ is an open interval containing $\lambda=0$, and $\left\{L_{\lambda}\right\}_{\lambda \in J}$ is an oriented family of Fredholm operators of index zero between them. Which one of the two orientations is chosen is not important for the detection of a sign jump at $\lambda=0$, the only point of $J$ where $L_{\lambda}$ is assumed singular.

We are concerned first with the case when $L_{\lambda}=L_{0}+\lambda K_{\lambda}$, where $K_{\lambda}$ : $E \rightarrow F$ is a family of linear operators which depend continuously on $\lambda \in J$. As a consequence of Theorem 4.6 we have the following result, in which a sort of Crandall-Rabinowitz condition for bifurcation is considered (see [4]).

Corollary 5.1. Let $L_{0} \in \Phi_{0}(E, F)$ and let $K_{\lambda}: E \rightarrow F(\lambda \in J)$ be a continuous family of linear operators. Assume that the following CrandallRabinowitz type condition is satisfied:

(CR) $u \in \operatorname{Ker} L_{0}$ and $K_{0} u \in \operatorname{Im} L_{0}$ imply $u=0$.

Then $L_{\lambda}=L_{0}+\lambda K_{\lambda}$ has a sign jump at $\lambda=0$ if and only if $\operatorname{Ker} L_{0}$ is odd dimensional.

Proof. Let $n=\operatorname{dim}\left(\operatorname{Ker} L_{0}\right)>0$ and let $P: F \rightarrow \operatorname{Im} L_{0}$ be any projection onto the closed subspace $F_{0}=\operatorname{Im} L_{0}$ of $F$. Let $S: \operatorname{coKer} L_{0} \rightarrow \mathbb{R}^{n}$ be 
any isomorphism and define $Q: F \rightarrow \mathbb{R}^{n}$ as the composition $Q=S R$, where $R: F \rightarrow$ coKer $L_{0}$ denotes the natural projection. In other words, consider any linear operator $Q$ onto $\mathbb{R}^{n}$ such that $\operatorname{Ker} Q=\operatorname{Im} L_{0}$.

Clearly, $P$ and $Q$ satisfy assumptions (4.1) and therefore, as observed in Remark 4.3, $P L_{0}$ is Fredholm of index $n$.

Let $A_{\lambda}: \mathbb{R}^{n} \rightarrow E$ be as in Lemma 4.5, with $M_{\lambda}=P L_{\lambda}$. By Theorem 4.6 it is enough to prove that $\operatorname{det}\left(Q L_{\lambda} A_{\lambda}\right)$ changes sign at $\lambda=0$ if and only if $n$ is odd. Since $Q L_{0}=0$, we have

$$
\operatorname{det}\left(Q\left(L_{0}+\lambda K_{\lambda}\right) A_{\lambda}\right)=\operatorname{det}\left(\lambda Q K_{\lambda} A_{\lambda}\right)=\lambda^{n} \operatorname{det}\left(Q K_{\lambda} A_{\lambda}\right) .
$$

Thus the assertion follows if we show that $\operatorname{det}\left(Q K_{\lambda} A_{\lambda}\right)$ is non-zero when $\lambda$ is small. This is true since assumption $(\mathrm{CR})$ means that the operator

$$
\left.R K_{0}\right|_{\text {Ker } L_{0}}: \operatorname{Ker} L_{0} \rightarrow \operatorname{coKer} L_{0}
$$

is injective and, consequently, $\operatorname{det}\left(Q K_{0} A_{0}\right) \neq 0$

A very special and interesting case of a family $L_{\lambda}$ is the one considered by Leray-Schauder in [12], where $L_{\lambda}=I-(\alpha+\lambda) K$, with $K: E \rightarrow E$ a compact linear operator and $L_{0}=I-\alpha K$ a singular operator (i.e., $\alpha^{-1}$ is an eigenvalue of $K$ ). They proved that the (Leray-Schauder) degree of $L_{\lambda}$ (which is defined for $\lambda$ in a pinched neighborhood of $0, \alpha^{-1}$ being isolated in the spectrum of $K)$ has a sign jump at $\lambda=0$ if and only if the algebraic multiplicity of $\alpha^{-1}$ is odd. We recall that the algebraic multiplicity of an eigenvalue $\mu \neq 0$ of $K$ is the dimension of the space $\cup_{k=1}^{\infty} \operatorname{Ker}(\mu I-K)^{k}$ which is well-known to be finite, since $K$ is compact.

Corollary 5.2 below, which will be deduced directly from Theorem 4.7, shows that for the above special family the same jump phenomenon occurs to the sign function. That is, $L_{\lambda}$ has a sign jump at $\lambda=0$ if and only if the algebraic multiplicity of $\alpha^{-1}$ is odd (hence, the same is true for the degree defined in [1]). Actually, in Corollary 5.2 a more general situation is considered, even if apparently different: the family $L_{\lambda}$ is of the type $L_{0}+\lambda I$, which formally does not include the case

$$
I-(\alpha+\lambda) K
$$

studied by Leray and Schauder. However, composing (5.1) with $(\alpha+\lambda)^{-1} I$ yields a new family which, as regards the possible existence of a sign jump, is equivalent to the previous one and, up to a reparametrization, is of the type $L_{0}+\lambda I$. We observe that in this (Leray-Schauder) case $L_{0}$ is a very special Fredholm operator of index 0; namely $L_{0}=\alpha^{-1} I-K$. 
Corollary 5.2. Let $L_{0}: E \rightarrow E$ be a non-invertible Fredholm operator of index 0. Assume that $\operatorname{dim}\left(\cup_{k=1}^{\infty} \operatorname{Ker} L_{0}^{k}\right)=n<\infty$. Then $L_{\lambda}=L_{0}+\lambda I$ has a sign jump at $\lambda=0$ if and only if $n$ is odd.

Proof. By assumption, there exists $p \in \mathbb{N}$ such that $\operatorname{Ker} L_{0}^{p+i}=\operatorname{Ker} L_{0}^{p}$ for all $i \in \mathbb{N}$. Put $E_{0}=\operatorname{Im} L_{0}^{p}$ and $E_{1}=\operatorname{Ker} L_{0}^{p}$. It is known that $E=E_{0} \oplus E_{1}$ (see, for example, [14: Chapter V, Theorem 6.2]). Clearly, one has $L_{0}\left(E_{0}\right) \subseteq$ $E_{0}$ and $L_{0}\left(E_{1}\right) \subseteq E_{1}$. Thus, $L_{\lambda}$ can be represented as a diagonal block-matrix as

$$
L_{\lambda}=\left(\begin{array}{cc}
T_{0}+\lambda I_{0} & 0 \\
0 & S_{0}+\lambda I_{1}
\end{array}\right)
$$

where $I_{0}$ is the identity of $E_{0}, I_{1}$ is the identity of $E_{1}$, and $T_{0}$ and $S_{0}$ are the restrictions of $L_{0}$ to $E_{0}$ and $E_{1}$, respectively. Observe now that $T_{0}: E_{0} \rightarrow E_{0}$ is Fredholm of index 0 and injective; therefore it is actually invertible. Thus, by Theorem $4.7, L_{\lambda}$ has a sign jump at $\lambda=0$ if and only if the same is true for the function $\lambda \mapsto \operatorname{det}\left(S_{0}+\lambda I_{1}\right)$. The assertion now follows from the fact that $\operatorname{det}\left(S_{0}+\lambda I_{1}\right)=\lambda^{n}$, since the spectrum $\sigma\left(S_{0}+\lambda I_{1}\right)$ of $S_{0}+\lambda I_{1}$ is given by $\{\lambda\}+\sigma\left(S_{0}\right)$, which coincides with the singleton $\{\lambda\}, S_{0}$ being nilpotent

Remark 5.3. Corollary 5.2 implies that, whenever $I-C \in \Psi_{K}(E)$ is an $I$-oriented automorphism, then $\operatorname{sign}(I-C)=(-1)^{p}$, where $p$ is the number of the eigenvalues of $C$ greater than 1 with odd multiplicity. Thus, by a well-known result of Leray and Schauder, we get

$$
\operatorname{sign}(I-C)=\operatorname{deg}_{L S}(I-C, B, 0)
$$

where $B$ is any open ball centered at the origin of $E$.

We now consider the situation in which one has a continuous family $M_{\lambda}$ of surjective Fredholm operators of index $n \in \mathbb{N}$ between two Banach spaces $G$ and $F$, and a surjective operator $C: G \rightarrow \mathbb{R}^{n}$. To understand how this situation can arise, we may think of $M_{\lambda}$ as a family of differential operators associated with a parameter-dependent differential equation, and of $C$ as expressing a set of boundary conditions (compare Examples 6.5 and 6.6 below).

The following result is a consequence of Theorem 4.6.

Corollary 5.4. Let $M_{\lambda}: G \rightarrow F$ be a continuous family of surjective Fredholm operators of index $n \in \mathbb{N}$, and let $A_{\lambda}: \mathbb{R}^{n} \rightarrow G$ be a continuous family of injective linear operators such that $\operatorname{Im} A_{\lambda}=\operatorname{Ker} M_{\lambda}$. Let $C: G \rightarrow$ $\mathbb{R}^{n}$ be linear and surjective, put $E=\operatorname{Ker} C$ and define $L_{\lambda}: E \rightarrow F$ by $L_{\lambda}=\left.M_{\lambda}\right|_{E}$. Then $L_{\lambda}$ has a sign jump at $\lambda=0$ if and only if the real function $\lambda \mapsto \operatorname{det}\left(C A_{\lambda}\right)$ changes sign crossing $\lambda=0$.

Proof. Define $N_{\lambda}: G \rightarrow F \times \mathbb{R}^{n}$ by $N_{\lambda} x=\left(M_{\lambda} x, C x\right)$. Theorems 2.4 and 2.5 imply that $N_{\lambda}$ is Fredholm of index 0 since $N_{\lambda}=S M_{\lambda}+K$, where $S$ is the inclusion of $F$ into $F \times \mathbb{R}^{n}$ and $K: G \rightarrow F \times \mathbb{R}^{n}$ is the map $x \mapsto(0, C x)$. 
Let $P$ and $Q$ denote the projections of $F \times \mathbb{R}^{n}$ onto the factors $F$ and $\mathbb{R}^{n}$, respectively. One can easily verify that $P$ and $Q$ satisfy properties (4.1), that $P N_{\lambda}=M_{\lambda}$ and that $Q N_{\lambda} A_{\lambda}=C A_{\lambda}$. Therefore, Theorem 4.6 implies that $N_{\lambda}$ has a sign jump at $\lambda=0$ if and only if the same is true for the real function $\operatorname{det}\left(C A_{\lambda}\right)$.

The assertion now follows from the fact that, by the reduction property of the $\operatorname{sign}\left(\right.$ Proposition 3.9), we have $\operatorname{sign}\left(L_{\lambda}\right)=\operatorname{sign}\left(N_{\lambda}\right)$ for any $\lambda \in J$. (Observe that the subspace $F \times\{0\}$ of $F \times \mathbb{R}^{n}$ is transverse to $N_{\lambda}$ and $N_{\lambda}^{-1}(F \times$ $\{0\})=E)$

\section{Examples}

We provide now some simple examples of ordinary differential operators in order to illustrate the meaning of the abstract results obtained in the previous sections.

Given a compact interval $[a, b]$, as usual $C^{0}([a, b])$, or simply $C([a, b])$, stands for the Banach space of the continuous real functions defined on $[a, b]$, with the norm

$$
\|x\|_{0}=\sup _{t \in[a, b]}|x(t)|
$$

of uniform convergence. More generally, given $n \in \mathbb{N}, C^{n}([a, b])$ denotes the Banach space of the $C^{n}$ real functions on $[a, b]$ with the norm

$$
\|x\|_{n}=\|x\|_{0}+\left\|x^{\prime}\right\|_{0}+\ldots+\left\|x^{(n)}\right\|_{0}
$$

or any equivalent norm ( $\operatorname{such}$ as $\left.\|x\|_{n}=\|x\|_{0}+\left\|x^{(n)}\right\|_{0}\right)$.

We now show how Theorem 4.6 can be applied in some concrete cases.

Example 6.1. Take $E=C^{2}([0, \pi])$ and $F=C^{0}([0, \pi]) \times \mathbb{R}^{2}$ and, for $\lambda$ in a neighborhood $J$ of 0 , consider the family of bounded linear operators $L_{\lambda}: E \rightarrow F$ given by

$$
L_{\lambda}(x)=(\ddot{x}+x+\lambda x, x(0), x(\pi)) .
$$

Clearly, given $\lambda$, the operator $L_{\lambda}$ is associated with the boundary value problem

$$
\left.\begin{array}{rl}
\ddot{x}+x+\lambda x & =y(t) \\
x(0) & =a \\
x(\pi) & =b
\end{array}\right\}
$$

where $y \in C^{0}([0, \pi])$ and $a, b \in \mathbb{R}$. 
Let us show first that $L_{\lambda}$ is Fredholm of index 0 . To this end we will use an argument that can be adapted to any (linear) boundary value problem for a linear ordinary differential equation (or system).

The differential operator

$$
M_{\lambda}: C^{2}([0, \pi]) \rightarrow C^{0}([0, \pi])
$$

defined by $M_{\lambda}(x)=\ddot{x}+x+\lambda x$, is onto with a two-dimensional kernel. Consequently, it is Fredholm of index 2. Thus, because of Theorem 2.5, the composition $C_{\lambda}=S M_{\lambda}$ of $M_{\lambda}$ with the natural inclusion

$$
S: C^{0}([0, \pi]) \rightarrow C^{0}([0, \pi]) \times \mathbb{R}^{2}
$$

is Fredholm of index zero. Now observe that $L_{\lambda}=C_{\lambda}+K$, with $K \in \mathrm{F}(E, F)$ given by $K(x)=(0, x(0), x(\pi))$, and apply Theorem 2.4 to show that $L_{\lambda} \in$ $\Phi_{0}(E, F)$.

With the notation of Theorem 4.6, put $F_{0}=C([0, \pi])$ and define $P: F \rightarrow$ $F_{0}$ by $P(y, a, b)=y$ and $Q: F \rightarrow \mathbb{R}^{2}$ by $Q(y, a, b)=(a, b)$. As already pointed out, the differential operator $M_{0}=P L_{0}$ is surjective and Fredholm of index 2. For $\lambda>-1$, the kernel of $P L_{\lambda}$ is the subspace of $C^{2}([0, \pi])$ spanned by the functions $u_{1}^{\lambda}(t)=\cos (\sqrt{1+\lambda} t)$ and $u_{2}^{\lambda}(t)=\sin (\sqrt{1+\lambda} t)$. Thus, for $\lambda$ small, we define $A_{\lambda}: \mathbb{R}^{2} \rightarrow E$ by

$$
\left(\alpha_{1}, \alpha_{2}\right) \mapsto \alpha_{1} u_{1}^{\lambda}+\alpha_{2} u_{2}^{\lambda}
$$

Hence, in (canonical) matrix form,

$$
Q L_{\lambda} A_{\lambda}=\left(\begin{array}{ll}
u_{1}^{\lambda}(0) & u_{2}^{\lambda}(0) \\
u_{1}^{\lambda}(\pi) & u_{2}^{\lambda}(\pi)
\end{array}\right)
$$

Therefore, by Theorem $4.6, L_{\lambda}$ has a sign jump at $\lambda=0$ since

$$
\operatorname{det}\left(Q L_{\lambda} A_{\lambda}\right)=\sin (\sqrt{1+\lambda} \pi) .
$$

Example 6.2. One may tackle the family $\left\{L_{\lambda}\right\}$ of Example 6.1 putting $F_{0}=C^{0}([0, \pi]) \times \mathbb{R}$ and defining the operators $P: F \rightarrow F_{0}$ and $Q: F \rightarrow \mathbb{R}$ by $P(y, a, b)=(y, a)$ and $Q(y, a, b)=b$. With this choice, the kernel of $P L_{\lambda}$ is one-dimensional and spanned by $u_{\lambda}(t)=\sin (\sqrt{1+\lambda} t)$. Therefore, given $\lambda$, one may define $A_{\lambda}: \mathbb{R} \rightarrow E$ by $A_{\lambda}(\alpha)=\alpha u_{\lambda}$. Thus, $\operatorname{det}\left(Q L_{\lambda} A_{\lambda}\right)=u_{\lambda}(\pi)$, and the same conclusion as in Example 6.1 follows.

The following two examples illustrate how Corollary 5.1 applies. 
Example 6.3. Take $E=\left\{x \in C^{2}([0, \pi]): x(0)=x(\pi)=0\right\}$ and $F=$ $C^{0}([0, \pi])$, and consider the family of bounded linear operators $L_{\lambda}: E \rightarrow F$ given by $L_{\lambda} x=\ddot{x}+x+\lambda x$. Clearly, these operators differ from those of Examples 6.1 and 6.2 but are related to the same boundary value problem. In fact, because of the surjectivity of the boundary operator $x \mapsto(x(0), x(\pi))$, this problem can be transformed into an equivalent one with the homogeneous boundary conditions $x(0)=0$ and $x(\pi)=0$.

Clearly, for any given $\lambda, L_{\lambda}$ is Fredholm of index zero, since it is the composition of the inclusion $S: E \rightarrow C^{2}([0, \pi])$ with the differential operator $M_{\lambda}: C^{2}([0, \pi]) \rightarrow C^{0}([0, \pi])$ defined by $M_{\lambda} x=\ddot{x}+x+\lambda x$ (see Theorem 2.5 ).

We can write $L_{\lambda}=L_{0}+\lambda K$, where $K$ is the inclusion of $E$ into $F$. In order to apply Corollary 5.1, we need to show that condition (CR) is satisfied.

Since $\operatorname{Ker} L_{0}$ is one-dimensional (precisely the vector space spanned by $u(t)=\sin t$ ), so is coKer $L_{0}$. Therefore, there exists a (unique up to a multiplicative constant) non-trivial linear functional $Q: F \rightarrow \mathbb{R}$ such that $\operatorname{Ker} Q=\operatorname{Im} L_{0}$. Let us try to find $Q$ of the type

$$
Q(y)=\int_{0}^{\pi} y(t) \varphi(t) d t
$$

where $\varphi$ is a (sufficiently regular) non-trivial function. Since $\operatorname{Im} L_{0}$ and $\operatorname{Ker} Q$ have the same (finite) codimension (which is one, in this case), any of the inclusions

$$
\operatorname{Im} L_{0} \subseteq \operatorname{Ker} Q \quad \text { or } \quad \operatorname{Im} L_{0} \supseteq \operatorname{Ker} Q
$$

implies the equality $\operatorname{Im} L_{0}=\operatorname{Ker} Q$. It is therefore sufficient to find $\varphi$ such that

$$
\int_{0}^{\pi}(\ddot{x}(t)+x(t)) \varphi(t) d t=0 \quad(x \in E)
$$

which means $\operatorname{Im} L_{0} \subseteq \operatorname{Ker} Q$. Integrating twice (6.1) by parts one can check that $\varphi(t)=\sin t$ satisfies this requirement. Thus, condition (CR) of Corollary 5.1 is verified since $\int_{0}^{\pi} \sin ^{2} t d t \neq 0$. Finally, since the dimension of Ker $L_{0}$ is odd, $L_{\lambda}$ has a sign jump at $\lambda=0$.

We point out that the existence of a sign jump of the differential operator $L_{\lambda}$ in Example 6.3 could be deduced directly from Example 6.1 using the reduction property of the sign (Proposition 3.9). In fact, this operator is the restriction to the pair of spaces

$$
\left\{x \in C^{2}([0, \pi]): x(0)=x(\pi)=0\right\} \quad \text { and } \quad C^{0}([0, \pi])
$$

of the other operator $L_{\lambda}$ in Example 6.2. Obviously, also the converse implication could be considered. 
The method used to deduce a sign jump of the differential operator $L_{\lambda}$ in Example 6.3 applies to a more general situation. Namely, to the case when the perturbation $\lambda K: E \rightarrow F$ is of the type $(\lambda K x)(t)=\lambda g(t) x(t)$ where $g$ is a continuous real function defined on $[0, \pi]$. In this case condition (CR) of Corollary 5.1 is fulfilled provided that $\int_{0}^{\pi} g(t) \sin ^{2} t d t \neq 0$.

The following example shows that for a periodic problem the sign jump, in some sense, is not likely to occur. Again we apply Corollary 5.1.

Example 6.4. Consider the Banach spaces

$$
\begin{aligned}
& E=\left\{x \in C^{2}([0,2 \pi]): x(0)=x(2 \pi), \dot{x}(0)=\dot{x}(2 \pi)\right\} \\
& F=C^{0}([0,2 \pi])
\end{aligned}
$$

and the family $L_{\lambda}: E \rightarrow F$ of bounded linear operators given by

$$
\left(L_{\lambda} x\right)(t)=\ddot{x}(t)+x(t)+\lambda g(t) x(t)
$$

where $g$ is a continuous function defined on $[0,2 \pi]$. Clearly, $L_{\lambda}$ is related to the problem with periodic boundary conditions

$$
\left.\begin{array}{rl}
\ddot{x}+x+\lambda g(t) x & =y(t) \\
x(0) & =x(2 \pi) \\
\dot{x}(0) & =\dot{x}(2 \pi)
\end{array}\right\} .
$$

As in the previous example, for any given $\lambda, L_{\lambda}$ is Fredholm of index zero, since it is the composition of the inclusion $S: E \rightarrow C^{2}([0,2 \pi])$ with the differential operator $M_{\lambda}: C^{2}([0,2 \pi]) \rightarrow C^{0}([0,2 \pi])$ defined by $M_{\lambda} x=$ $\ddot{x}+x+\lambda g x$.

Denote by $L_{0}$ and $K$ the operators (from $E$ to $F$ ) defined by $L_{0} x=\ddot{x}+x$ and $K x=g x$, so that $L_{\lambda}=L_{0}+\lambda K$. In order to apply Corollary 5.1, we need to determine the spaces $\operatorname{Ker} L_{0}$ and $\operatorname{Im} L_{0}$. The subspace $\operatorname{Ker} L_{0}$ of $E$ contains the functions $u_{1}(t)=\cos t$ and $u_{2}(t)=\sin t$, thus it is twodimensional ( $L_{0}$ being a second order differential operator). Consequently, $\operatorname{Im} L_{0}$ has codimension two. With the same argument as in Example 6.3, one can check that $y$ belongs to $\operatorname{Im} L_{0}$ only if (and consequently if)

$$
\int_{0}^{2 \pi} y(t) \cos t d t=\int_{0}^{2 \pi} y(t) \sin t d t=0 .
$$

Thus, if the determinant of the matrix

$$
\left(\begin{array}{cc}
\int_{0}^{2 \pi} g(t) \cos ^{2} t d t & \int_{0}^{2 \pi} g(t) \sin t \cos t d t \\
\int_{0}^{2 \pi} g(t) \cos t \sin t d t & \int_{0}^{2 \pi} g(t) \sin ^{2} t d t
\end{array}\right)
$$


is different from zero, condition (CR) of Corollary 5.1 is verified. Hence, in this case, $L_{\lambda}$ has not a sign jump at $\lambda=0$, the dimension of Ker $L_{0}$ being even.

As regards the above example, and with the same notation, if the determinant of the matrix (6.2) is zero (e.g. when $g(t)=1+2 \sin 2 t$ ), Corollary 5.1 does not apply. In this case, to verify whether $L_{\lambda}$ has a sign jump at $\lambda=0$ one can use another consequence of Theorem 4.6; namely, Corollary 5.4. In order to apply this result it is not necessary to determine $\operatorname{Im} L_{0}$, but it is not sufficient to find out just $\operatorname{Ker} L_{0}$ : we actually need to evaluate $\operatorname{Ker} M_{\lambda}$ for $\lambda$ in a neighborhood of 0 . This can be very hard with pencil and paper, and in most cases impossible. Numerical computations, however, may help to overcome these difficulties, as shown in the following example.

Example 6.5. Consider the Banach spaces

$$
\begin{aligned}
& G=C^{2}([0,2 \pi]) \\
& E=\{x \in G: x(0)=x(2 \pi), \dot{x}(0)=\dot{x}(2 \pi)\} \\
& F=C^{0}([0,2 \pi])
\end{aligned}
$$

and the family $L_{\lambda}: E \rightarrow F$ given by the restriction to $E$ of the differential operators $M_{\lambda}: G \rightarrow F$ defined as

$$
\left(M_{\lambda} x\right)(t)=\ddot{x}(t)+x(t)+\lambda(1+2 \sin 2 t) x(t) .
$$

To detect a possible sign jump of $L_{\lambda}$, the method employed in Example 6.4 does not work in this case, since the determinant of matrix (6.2) is zero when $g(t)=1+2 \sin 2 t$. However, we will use Corollary 5.4, in combination with numerical computations, to verify that $L_{\lambda}$ has a sign jump at $\lambda=0$. Here, the boundary operator $C: G \rightarrow \mathbb{R}^{2}$ is given by

$$
C x=(x(2 \pi)-x(0), \dot{x}(2 \pi)-\dot{x}(0)) .
$$

Given $\lambda$ in a neighborhood of 0 , let $u_{1}^{\lambda}$ and $u_{2}^{\lambda}$ denote the solutions of the differential equation

$$
\ddot{x}(t)+x(t)+\lambda(1+2 \sin 2 t) x(t)=0
$$

satisfying the Cauchy conditions $u_{1}^{\lambda}(0)=1, \dot{u}_{1}^{\lambda}(0)=0$ and $u_{2}^{\lambda}(0)=0, \dot{u}_{2}^{\lambda}(0)=$ 1. Thus, for $\lambda$ small, the kernel of $M_{\lambda}$ is spanned by $u_{1}^{\lambda}$ and $u_{2}^{\lambda}$, and a continuous family of injective operators $A_{\lambda}: \mathbb{R}^{2} \rightarrow G$ such that $\operatorname{Im} A_{\lambda}=$ Ker $M_{\lambda}$ is, for example, $A_{\lambda}(\alpha, \beta)=\alpha u_{1}^{\lambda}+\beta u_{2}^{\lambda}$. With this choice of $A_{\lambda}$, the real function $\sigma(\lambda)=\operatorname{det}\left(C A_{\lambda}\right)$ as in Corollary 5.4 is

$$
\sigma(\lambda)=\operatorname{det}\left(\begin{array}{ll}
u_{1}^{\lambda}(2 \pi)-u_{1}^{\lambda}(0) & u_{2}^{\lambda}(2 \pi)-u_{2}^{\lambda}(0) \\
\dot{u}_{1}^{\lambda}(2 \pi)-\dot{u}_{1}^{\lambda}(0) & \dot{u}_{2}^{\lambda}(2 \pi)-\dot{u}_{2}^{\lambda}(0)
\end{array}\right)
$$


and a computer analysis of this function by a standard numerical ODE-solver shows that $\sigma(\lambda)$ changes sign at $\lambda=0$. Thus, as claimed, $L_{\lambda}$ has a sign jump at $\lambda=0$.

The method used in Example 6.5 to detect a sign jump can be extended in order to include any linear boundary value problem for a family of first order differential systems. We close with the following abstract example, which illustrates this extension. $\mathbb{R}^{n}$

Example 6.6. Consider the family of ordinary differential equations in

$$
\dot{x}(t)+(A(t)+\lambda B(t)) x(t)=y(t) \quad(\lambda \in \mathbb{R})
$$

where $A$ and $B$ are $n \times n$ matrices of continuous functions defined on a compact interval $[a, b]$, the assigned function $y:[a, b] \rightarrow \mathbb{R}^{n}$ is continuous, and the unknown function $x:[a, b] \rightarrow \mathbb{R}^{n}$ is of class $C^{1}$. Let $G$ and $F$ denote, respectively, the Banach spaces

$$
C^{1}\left([a, b], \mathbb{R}^{n}\right) \cong\left(C^{1}([a, b])\right)^{n} \quad \text { and } \quad C^{0}\left([a, b], \mathbb{R}^{n}\right) \cong\left(C^{0}([a, b])\right)^{n} .
$$

Associated with (6.3) one has the operators $M_{\lambda}: G \rightarrow F$ defined by

$$
\left(M_{\lambda} x\right)(t)=\dot{x}(t)+(A(t)+\lambda B(t)) x(t)
$$

which are surjective with $n$-dimensional kernel (because of well-known results on differential equations). Let $C x=0$ be a (well-posed) linear boundary condition for (6.3); that is, $C$ is a surjective linear operator from $G$ to $\mathbb{R}^{n}$ (so that $\operatorname{Ker} C$ has codimension $n$ in $G$ ). Put $E=\operatorname{Ker} C$ and define the family of differential operators $L_{\lambda}: E \rightarrow F$ by the restriction of $M_{\lambda}$ to $E$. The operators $L_{\lambda}$ are Fredholm of index 0 , as composition of the inclusion $S: E \rightarrow G$ (whose index is $-n$ ) with $M_{\lambda}$ (whose index is $n$ ).

Assuming that $L_{0}$ is singular, to detect a possible sign jump for $L_{\lambda}$ (at $\lambda=0)$ we proceed as follows. Let $\left\{e_{1}, e_{2}, \ldots, e_{n}\right\}$ be the canonical basis of $\mathbb{R}^{n}$. For any $i \in\{1,2, \ldots, n\}$ let $u_{i}^{\lambda}$ denote the (maximal) solution of equation (6.3) satisfying the Cauchy condition $u_{i}^{\lambda}(a)=e_{i}$. Clearly, because of the linearity of equation (6.3), any $u_{i}^{\lambda}$ is defined on the whole interval $[a, b]$. Notice that, given $\lambda$, the matrix $Y_{\lambda}(t)$ whose columns are the solutions $u_{i}^{\lambda}(t)$ is a fundamental matrix of system (6.3). Denote by $C Y_{\lambda}$ the $n \times n$ real matrix whose columns are the vectors $C u_{i}^{\lambda}$. As in Example 6.5, Corollary 5.4 implies that if the function $\sigma(\lambda)=\operatorname{det}\left(C Y_{\lambda}\right)$ has a sign jump at $\lambda=0$, the same is true for the family $L_{\lambda}$. 


\section{References}

[1] Benevieri, P. and M. Furi: A simple notion of orientability for Fredholm maps of index zero between Banach manifolds and degree theory. Ann. Sci. Math. Québec 22 (1998), 131 - 148.

[2] Benevieri, P. and M. Furi: On the concept of orientability for Fredholm maps between real Banach manifolds. Top. Methods Nonlin. Anal. 16 (2000), 279 306.

[3] Benevieri, P. and M. Furi: Bifurcation results for families of Fredholm maps of index zero between Banach spaces. Nonlin. Anal. Forum 6 (2001), 35 - 47.

[4] Crandall, M. G. and P. H. Rabinovitz: Bifurcation from simple eigenvalues. J. Funct. Anal. 8 (1971), $321-340$.

[5] Dold, A.: Lectures on Algebraic Topology. Berlin: Springer-Verlag 1973.

[6] Fitzpatrick, P. M. and J. Pejsachowicz: Parity and generalized multiplicity. Trans. Amer. Math. Soc. 326 (1991), 281 - 305.

[7] Fitzpatrick, P. M. and J. Pejsachowicz: Orientation and the Leray-Schauder theory for fully nonlinear elliptic boundary value problems. Mem. Amer. Math. Soc. 483 (1993).

[8] Fitzpatrick, P. M., Pejsachowicz, J. and P. J. Rabier: The degree of proper $\mathrm{C}^{2}$ Fredholm mappings. J. reine angew. Math. 427 (1992), 1 - 33.

[9] Fitzpatrick, P. M., Pejsachowicz, J. and P. J. Rabier: Orientability of Fredholm families and topological degree for orientable nonlinear Fredholm mappings. J. Funct. Anal. 124 (1994), 1 - 39.

[10] Kirillov, A. A. and A. D. Gvishiani: Theorems and Problems in Functional Analysis. New York: Springer-Verlag 1982.

[11] Kuiper, N. H.: The homotopy type of the unitary group of a Hilbert space. Topology 3 (1965), 19 - 30.

[12] Leray, J. and J. Schauder: Topologie et équations fonctionnelles. Ann. Sci. École Norm. Sup. 51 (1934), 45 - 78.

[13] Pejsachowicz, J. and P. J. Rabier: Degree theory for $C^{1}$ Fredholm mappings of index 0. J. Anal. Math. 76 (1998), 289 - 319.

[14] Taylor, A. E. and D. C. Lay: Introduction to Functional Analysis. New York et al.: John Wiley \& Sons 1980.

Received 04.08.2002 